\title{
Hemorrhagic pustules in an Aboriginal man
}

\section{Kerry MacDonald MD, Philippe Lagacé-Wiens MD, John Embil MD}

$\mathrm{A}$ 68-year-old Aboriginal man who lived in northwestern Ontario presented to a local hospital with back pain and fever. Over the past several days, crops of vesicopustular lesions, primarily affecting his extremities, had developed. The lesions gradually became hemorrhagic and tender. He was unaware of anyone with a similar illness. He had no known exposure to tuberculosis or animals, and he had not travelled outside of Ontario. His medical history included remote traumatic brain injury, alcohol abuse, cigarette smoking, chronic obstructive pulmonary disease, hypertension, type 2 diabetes mellitus (managed with diet alone) and recent cellulitis in his right arm with right brachial vein thrombosis. He reported no history of intravenous drug use. When his current symptoms had begun, he was receiving treatment for cellulitis and thrombosis, and he had been living in a medical hostel for the purpose of monitoring warfarin therapy.

At the time of presentation, a physical examination revealed crops of hemorrhagic vesicles primarily affecting the patient's limbs (Figure 1). These lesions were tender and had an erythematous base. Hemorrhagic and purulent material could be expressed from the vesicles. There was no cardiac murmur, but diffuse crackles were heard throughout all lung fields.

Two days after admission, the patient reported that he had experienced progressive shortness of breath in the days leading to the presentation. A chest radiograph showed normal cardiac size and no pulmonary infiltrates, effusions or masses. An echocardiogram showed normal valves without vegetations. His leukocyte count was 14.0 (normal 3.5-10.5) $\times 10^{9} / \mathrm{L}$, and his neutrophil count was 12.1 (normal $2.5-6.0) \times 10^{9} / \mathrm{L}$ with no left shift. His international normalized ratio (INR) was considerably elevated at 7.0 (normal 0.91.1) because of warfarin use. The patient's liver and renal profiles were normal. Blood cultures, started at the time of presentation and repeated during his stay in hospital, were all sterile.

Four days after admission, respiratory distress developed. The patient required intubation and mechanical ventilation seven days after admission. A chest radiograph obtained six days after admission showed interstitial and airspace disease with air bronchograms in the right upper and both left and right mid-lung fields (Figure 2).

\section{Which condition is not in the initial differential diagnosis?}
a. Disseminated varicella-zoster virus
b. Disseminated meningococcal infection
c. Pyoderma gangrenosum
d. Staphylococcal endocarditis
e. Bullous pemphigoid

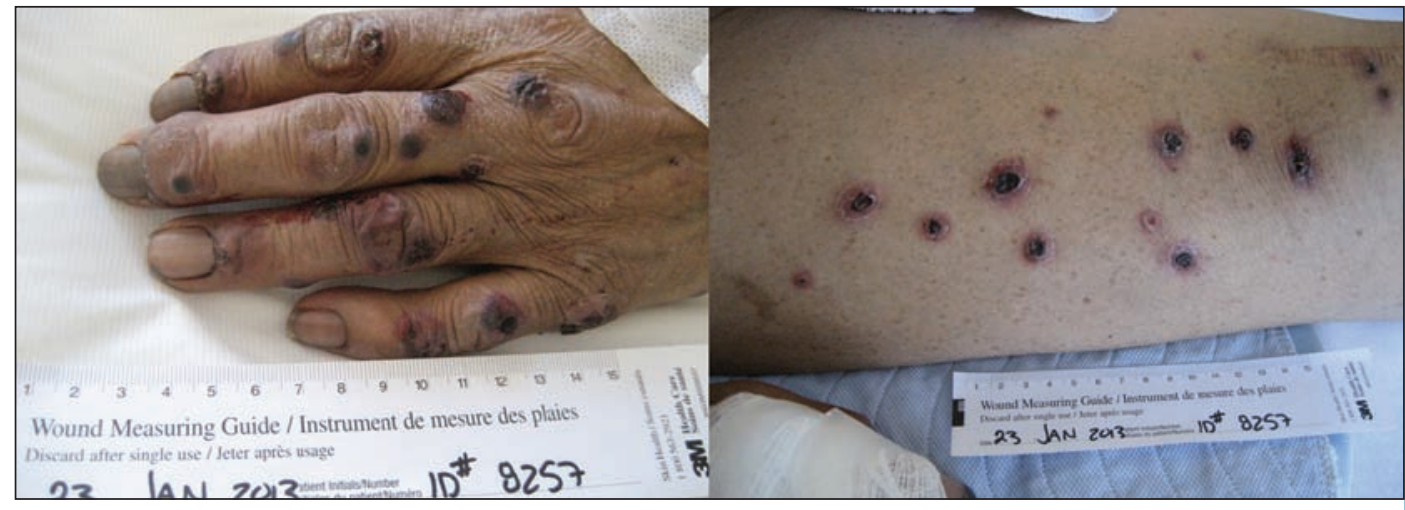

Figure 1: Hemorrhagic vesicular lesions on the hands and legs of a 68-year-old man. Most lesions were vesicular and 5-10 $\mathrm{mm}$ in diameter, and some were ulcerated. Circumlesional erythema was present on most lesions. Vesicular lesions were fluctuant and filled with purulent hemorrhagic material.
Competing interests: None declared.

This article has been peer reviewed.

\section{Correspondence to:}

Philippe Lagacé-Wiens, plagacewiens@dsmanitoba .ca

CMAJ 2014. DOI:10.1503 /cmaj.131328 
The answer is (e). Bullous pemphigoid lesions are not purulent and are generally clear or serous fluidfilled vesicles larger than $10 \mathrm{~mm}$ in diameter. Bullous pemphigoid may be associated with neurologic disorders or malignant disease, but it is not generally seen in association with diffuse lung disease. ${ }^{1}$ Pustular pyoderma gangrenosum is typically seen in patients with underlying autoimmune disorders, most commonly inflammatory bowel disease; this condition may also be seen in patients with rheumatoid arthritis and vasculitis, which

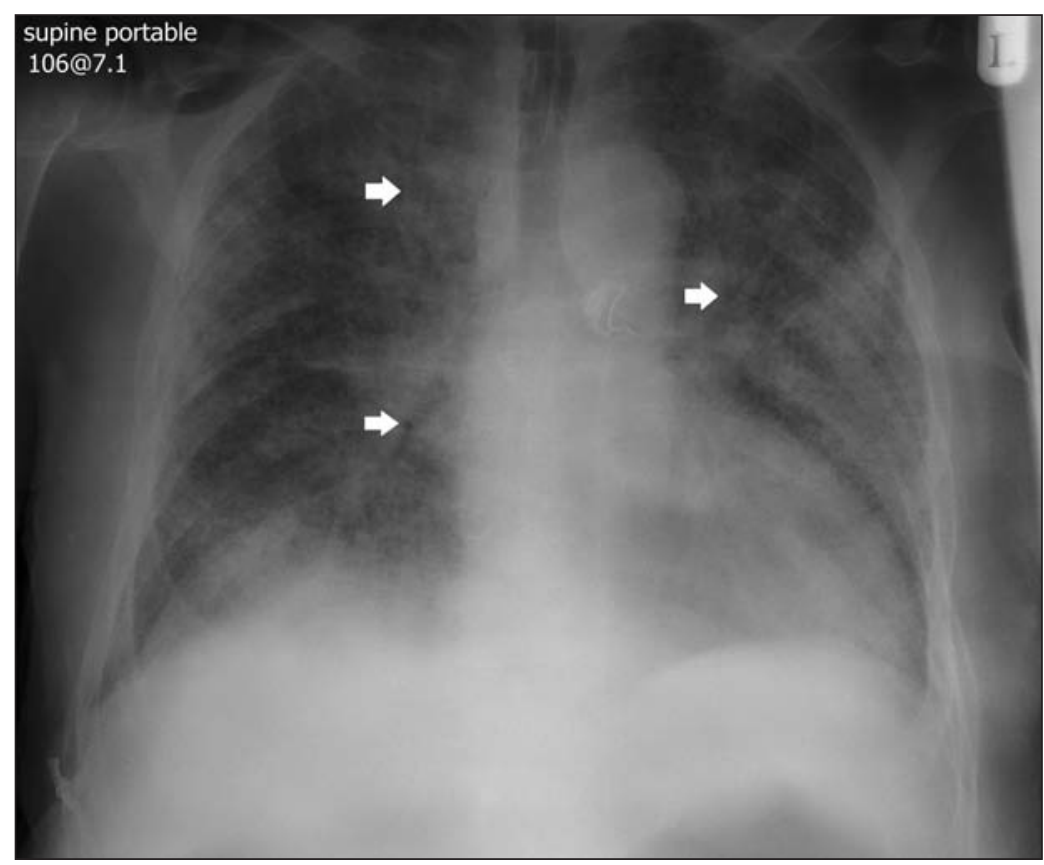

Figure 2: Anterior-posterior radiograph of the patient's chest obtained six days after admission. Diffuse interstitial and airspace disease with air bronchograms (arrows) can be seen in the right upper and both left and right mid-lung fields.

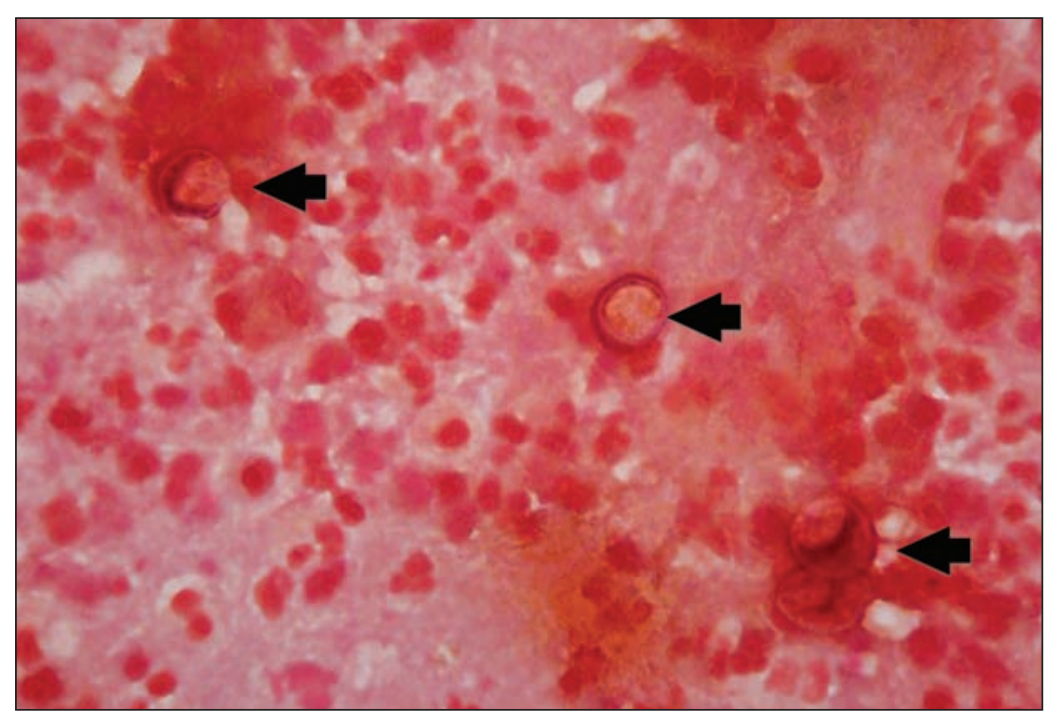

Figure 3: Gram staining of vesicle fluid (original magnification $\times 1000$ ). Polymorphonuclear cells can be seen in the background. The presence of characteristic spherical, poorly stained structures (diameter about 8-15 $\mu \mathrm{m}$; arrows) confirmed the diagnosis. could potentially lead to lung disease. ${ }^{2}$ Disseminated varicella-zoster virus infection, meningococcemia and staphylococcal endocarditis could all be associated with lung and skin findings.

\section{What is the most appropriate diagnostic intervention at this time?}

a. Varicella-zoster virus immunoglobulin $G$ (IgG) titres

b. Aspirate of a pustule for microscopy and culture

c. Rheumatoid factor levels

d. Sputum cultures

e. Transesophageal echocardiogram

The answer is (b). A single varicella-zoster virus IgG titre would not be useful in cases of suspected acute disease. ${ }^{3}$ Determining the level of rheumatoid factor and obtaining sputum cultures or an echocardiogram may be important adjunct investigations, but they are unlikely to provide a definitive diagnosis in isolation. ${ }^{4}$ An aspirate of the lesions was used to determine the diagnosis (Figure 3).

\section{What is the diagnosis?}

a. Disseminated cryptococcosis

b. Disseminated blastomycosis

c. Staphylococcus aureus pyoderma (due to underlying endocarditis)

d. Disseminated varicella-zoster virus infection (with varicella-zoster virus pneumonia)

e. Secondary syphilis

The answer is (b). The broad-based, thick-walled yeast cells visible in the gram stain are characteristic of Blastomyces dermatitidis. The observation of these characteristic forms confirmed the diagnosis of disseminated blastomycosis ${ }^{4}$ in this patient. The fungal culture ultimately grew $B$. dermatitidis, confirming the diagnosis. Although $B$. dermatitidis can be seen on gramstained infected material, the recognition of the typical structure requires expertise and experience. Clinicians should be aware that a specific request should be made for fungal stains (e.g., calcofluor) and fungal culture of aspirates or tissue biopsies when blastomycosis is suspected. Swabs should not be submitted because the quantity of the material present is frequently insufficient to make the microbiologic diagnosis.

\section{Discussion}

Blastomycosis is a systemic fungal disease caused by the thermally dimorphic fungus $B$. dermatitidis. ${ }^{4,5}$ Although uncommon, the disease 
is endemic to the Ohio and Mississippi River basins and in the Canadian provinces and American states that border the Great Lakes, and is hyperendemic in the area of northwestern Ontario surrounding Lake of the Woods (Kenora, Ontario). ${ }^{5}$ In this region, retrospective epidemiologic analysis has found that the symptomatic incidence rate is 7.11 cases per 100000 people, or about five times the reported incidence rate in Mississipi and Wisconsin (1.3 and 1.4 cases per 100000 people, respectively). ${ }^{5,6}$

Blastomycosis is acquired by the inadvertent inhalation of conidia; these are produced by the mycelial form of the fungus, which exists in nature. ${ }^{5.7}$ The infectious conidia are transformed in the lungs into the yeast form, causing clinical blastomycosis if the infection is not controlled by the cell-mediated immune response. ${ }^{4.5}$ Disease onset may be acute, occurring shortly after exposure, or it may be the result of reactivation following a remote exposure. ${ }^{4,5}$ An outbreak investigation in Wisconsin found that infection rates following exposure appear to be high but that fewer than $50 \%$ of infected individuals develop clinically apparent disease. ${ }^{5}$

Retrospective observational studies suggest that, in most individuals, the respiratory tract is the primary site of disease, and the clinical presentation may be acute, subacute or chronic. ${ }^{4-8}$ Several epidemiologic surveys have confirmed that disseminated disease, which may occur concurrently or subsequently to respiratory disease, occurs in $25 \%-30 \%$ of symptomatic individuals. ${ }^{4-6}$ Disseminated disease most commonly involves (in order of frequency) the skin, bone, the genitourinary tract and the central nervous system. ${ }^{4,5,8}$ Skin involvement occurs in $40 \%-80 \%$ of cases of disseminated blastomycosis. Presentation is typically one or a few papulopustular or verrucous lesions (so-called papulopustular cutaneous blastomycosis) or one or few ulcerated lesions (so-called ulcerative cutaneous blastomycosis). Lesions typically appear granulomatous on histopathologic examination. ${ }^{4}$

This patient's presentation was unusual because of the widespread vesicopurulent cutaneous findings. The purulent and vesicular lesions appeared in crops, which is more characteristic of varicella-zoster virus infection, ${ }^{3}$ and constitutes a rare form of disseminated cutaneous blastomycosis (only seven reported cases). ${ }^{8-12}$ Most reported cases of pustular blastomycosis (five of seven cases) occurred in Tennessee, where it has been postulated that a genetic variant of $B$. dermatitidis may be the cause of this unusual form of the disease. ${ }^{8}$ In our patient, the use of warfarin and his high INR likely contributed to the hemorrhagic appearance of what may have initially been pus- tular lesions. The hemorrhagic nature of these disseminated lesions in an older patient also raised the possibility of encapsulated bacterial pathogens, such as Haemophilus influenzae, Neisseria meningitidis and Streptococcus pneumoniae. However, multiple blood cultures were sterile, and the clinical presentation was not the typically acute manifestation associated with bacterial sepsis. In light of the concomitant pulmonary disease and chest radiographic findings, it is likely that this patient had concurrent pulmonary and disseminated cutaneous blastomycosis. The evidence of consolidation on the chest radiograph suggests the possibility of acute pulmonary disease and early dissemination rather than reactivation.

\section{Assuming that the patient has no contraindications, what treatment should be initially provided?}

\author{
a. Amphotericin B \\ b. Fluconazole \\ c. Terbinafine \\ d. Caspofungin (or micafungin) \\ e. Itraconazole
}

The correct answer is (a). Current treatment for moderately severe to severe disseminated blastomycosis should include amphotericin B..$^{13}$ Guidelines by the Infectious Diseases Society of America recommend (based on descriptive studies, case reports and expert opinion) the intravenous use of amphotericin B lipid formulation (3$5 \mathrm{mg} / \mathrm{kg}$ daily) or amphotericin B deoxycholate $(0.7-1 \mathrm{mg} / \mathrm{kg}$ daily) for one to two weeks until stable followed by a 12-month course of itraconazole given orally. ${ }^{13}$ However, the recognized renal toxicity and risk of hypotension, hypokalemia and arrhythmia may limit the use of amphotericin $\mathrm{B}$ even in cases of severe disease. In such cases, alternative agents such as itraconazole, high-dose fluconazole or voriconazole may need to be considered, recognizing that there is little evidence to support their use in severe disease. ${ }^{13}$ For mild to moderate disease, evidence from case-control series suggests that itraconazole alone can be prescribed and that treatment should last 612 months. ${ }^{13}$ Echinocandins are not recommended for the treatment of blastomycosis. ${ }^{13}$

Our patient was initially given itraconazole; this was changed to amphotericin B lipid complex and methlyprednisone after intubation, and his condition rapidly improved. Ventilator support was stopped three days after intubation, and the patient was discharged from the intensive care unit to complete treatment, and rehabilitate 
and await placement. Results of additional investigations for immunocompromise (including serologic testing for HIV), hematologic malignant disease and autoimmune disorders were negative.

\section{Conclusion}

Blastomycosis is uncommon in Canada, but exposure to specific geographic regions (e.g., northwestern Ontario and the Mississippi valley) should prompt clinicians to consider this disease. Although pulmonary disease is the most common manifestation, cutaneous disease is the second most common form of blastomycosis and may occur concurrently with pulmonary disease. Diagnosis requires a high degree of clinical suspicion and appropriate samples (respiratory samples for pulmonary disease; aspirates or biopsies for cutaneous disease) submitted for fungal stains and culture. Management depends on the severity of disease but invariably requires longterm antifungal treatment.

\section{References}

1. Schmidt E. Pemphigoid diseases. Lancet 2013;381:320-32.

2. Ahronowitz I, Harp J, Shinkai K. Etiology and management of pyoderma gangrenosum: a comprehensive review. Am J Clin Dermatol 2012;13:191-211.

3. Arvin AM. Varicella-zoster virus. Clin Microbiol Rev 1996;9: 361-81.

4. Saccente M, Woods GL. Clinical and laboratory update on blastomycosis. Clin Microbiol Rev 2010;23:367-81.

5. Crampton TL, Light RB, Berg GM, et al. Epidemiology and clinical spectrum of blastomycosis diagnosed at Manitoba hospitals. Clin Infect Dis 2002;34:1310-6.

6. Chapman SW, Lin AC, Hendricks KA, et al. Endemic blastomycosis in Mississippi: epidemiological and clinical studies. Semin Respir Infect 1997;12:219-28.

7. Klein BS, Vergeront JM, Weeks RJ, et al. Isolation of Blastomyces dermatitidis in soil associated with a large outbreak of blastomycosis in Wisconsin. N Engl J Med 1986;314:529-34.

8. Fisher KR, Baselski V, Beard G, et al. Pustular blastomycosis. J Am Acad Dermatol 2009;61:355-8.

9. Sweeney EW, Franks A Jr, Silva-Hunter M. An unusual case of North American blastomycosis in New Jersey: its clinical course and response to amphotericin B. Cutis 1982;30:199-202,204-6.

10. Khakoo R, Roidad N. Vesico-pustular blastomycosis. Int J Infect Dis 2012;16:e763-4.

11. Hashimoto K, Kaplan RJ, Daman LA, et al. Pustular blastomycosis. Int J Dermatol 1977; 16:277-80.

12. Friedman R, Henson T, Skinner R. Pustular eruption. Arch Dermatol 2002; 138:1371-6.

13. Chapman SW, Dismukes WE, Proia LA, et al. Clinical practice guidelines for the management of blastomycosis: 2008 update by the Infectious Diseases Society of America. Clin Infect Dis 2008; 46:1801-12.

Affiliations: Department of Pathology (MacDonald), Lake of the Woods District Hospital, Kenora, Ont.; Department of Medical Microbiology and Infectious Diseases (LagacéWiens, Embil), University of Manitoba, Winnipeg, Man.; Department of Clinical Microbiology (Lagacé-Wiens), Diagnostic Services Manitoba, Saint-Boniface Hospital, Winnipeg, Man.; Infectious Diseases Section, Department of Medicine (Embil), University of Manitoba, Winnipeg, Man.

Contributors: All of the authors contributed substantially to the writing and revising of the manuscript and approved the final version submitted for publication. 